Original Article

\title{
Difference in contributions to the team's score in female wheelchair basketball at the 2016 Rio Paralympics by physical capability classification
}

\author{
Takashi Doi ${ }^{1) *}$, Hirotaka Mutsuzaki, MD, PhD ${ }^{2)}$, Kaori Tachibana, RPT, PhD ${ }^{3)}$, \\ Yasuyoshi Wadano, MD, PhD ${ }^{1)}$, KoIChi Iwai, PhD $^{4)}$ \\ 1) Center for Medical Sciences, Ibaraki Prefectural University of Health Sciences: 4669-2 Ami, \\ Ami-machi, Inashiki-gun, Ibaraki 300-0394, Japan \\ 2) Department of Orthopaedic Surgery, Ibaraki Prefectural University of Health Sciences, Japan \\ 3) Department of Physical Therapy, Ibaraki Prefectural University of Health Sciences, Japan \\ 4) Center for Humanities and Sciences, Ibaraki Prefectural University of Health Sciences, Japan
}

\begin{abstract}
Purpose] This study clarified differences in players' contributions to the team's score in female wheelchair basketball at the 2016 Rio Paralympics by physical capacity classification, and examined the roles required in the team. [Subjects and Methods] This study used stats (record of play contents) for players who played for more than 20 minutes from the official box scores of all 31 games at the 2016 Paralympics. Players were divided into three groups by physical capacity classification: low, middle, and high. The average stats for each group were compared and the covariance structure was analyzed to determine the role of each group during the game. [Results] Comparisons showed that the higher the class, the higher the value of many stats items. Important elements were defensive rebound, steal, and turnover in the low group; and score, offensive rebound, and turnover in the high group. [Conclusion] Players in the high group have more plays related to the ball. Those in the low group should increase the numbers of steals and defensive rebounds and reduce turnover. High group players are required to have scoring ability, acquire offensive rebound, and reduce turnover.

Key words: Female wheelchair basketball, Classification, Contribution to scoring
\end{abstract}

(This article was submitted Nov. 20, 2017, and was accepted Jan. 9, 2018)

\section{INTRODUCTION}

Wheelchair basketball rules are similar to those of basketball, but differ in terms of the classification system. In wheelchair basketball, players are grouped into eight classes (categories), based on their physical capacity to execute fundamental basketball movements ${ }^{1}$. The International Wheelchair Basketball Federation (IWBF) awards players points from 1.0 (minimal physical capacity) to 4.5 (maximal physical capacity); the total points of all players on the court must not exceed 14 . This classification depends on differences in trunk function. Several studies have compared the performance between classes ${ }^{2-4)}$, but focused on the superiority and inferiority of performance using scoring ability, or scoring each stats (record of play contents) item and basing evaluation on the sum of these items. A player's ability to shoot is important, as basketball is a competition for the highest score. However, it is difficult to create a situation in which a player can easily shoot by themselves. Cooperation of all five players is necessary to make open shots; not all members are required to score as the team's score is important rather than individual scores. Therefore, the importance of each stats item differs depending on the role played by each player in scoring for the team. In basketball, several studies have been conducted to clarify differences in game performance by position and important stats items ${ }^{5-7)}$. When players with different functional abilities are mixed on the

*Corresponding author. Takashi Doi (E-mail: doita@ipu.ac.jp)

(C2018 The Society of Physical Therapy Science. Published by IPEC Inc.

(c) (i) $(-)$ This is an open-access article distributed under the terms of the Creative Commons Attribution Non-Commercial No Derivacc. 
court (as they are in wheelchair basketball), the role of players in each class becomes even more important. However, the role of players by physical capacity class has not been investigated. This study examined how each class contributed to the team's score in female wheelchair basketball at the 2016 Rio Paralympic Games, and the role required for each class in the team.

\section{SUBJECTS AND METHODS}

Data were drawn from the box scores of all 31 female wheelchair basketball games at the 2016 Rio Paralympics. The Human Ethics Review Committee of Ibaraki Prefectural University of Health Sciences approved this study (approval No. e132). This included the team's total score (TS) and players' personal stats. Ten countries participated: the United States, Germany, the Netherlands, the United Kingdom, Canada, China, Brazil, France, Argentina, and Algeria. Individual stats items drawn from the box score were: playing time (PT); points (PTS); field goal made (FGM) or shots excluding free throw success; field goal attempts (FGA); field goal percentage (FG\%); free throw made (FTM) or free throw made during a shooting attempt following an awarded foul; free throw attempts (FTA); free throw percentage (FT\%); offensive rebound (OR) or receiving the rebound ball from a failed shooting attempt from own team; defensive rebound (DR) or receiving the rebound ball from a failed opposition shooting attempt; assist (AS) or assisting shot success by passing; turnover (TO) or ball lost by mistake; steal (ST) or stealing the ball from opponents; block shot (BS); personal foul (PF) or fouling an opponent; and draw a foul (FD) or receiving a foul.

Based on Sampaio et al. ${ }^{5}$, we analyzed the stats of 297 players with a game duration of more than 20 minutes each. In accordance with IWBF classification points, players' whose stats were obtained were classified into three groups: low (1.0-2.0 points, 124 stats), middle (2.5-3.5 points, 88 stats), and high (4.0-4.5 points, 85 stats). Features of trunk function in each group were: the low group had little or no controlled trunk movement; the middle group had almost complete trunk movement in the forward plane, but no controlled movement in the sideways plane; and the high group could use their trunk almost freely.

We conducted one-way analysis of variance to investigate differences in each stats item among the three groups using SPSS version 24 . We also created models of how each group contributed to the score by covariance structure analysis using AMOS version 25. Observational variables used in covariance structure analysis were TS and individual stats (FGM, FGA, FTM, FTA, OR, DR, AS, TO, ST, BS, PF, FD), which were directly counted during play. These items were divided into three major categories: 1) items directly connected to the score (FGM, FGA, FTM, FTA, FD), 2) items to increase score opportunities (OR, DR, ST, BS), and 3) items to reduce opponents' score opportunities (TO, PF). Category 1 included FD, as a free throw does not occur unless a foul is received, in addition to the shoot/assist items which directly add to the score. Categories 2 and 3 were included in the models if the path coefficients between TS and observation variables showed significant differences in the Wald test. And they were also included if the path coefficients between extracted item and other observation variables showed significant differences. Category 1 is directly linked to TS and was included in the models regardless of significant difference. However, since FD overlaps with FTA, the path coefficient with TS was not calculated. Significance was set at $\mathrm{p}<0.05$. Model fit was assessed using the comparative fit index (CFI), Root Mean Square Error of Approximation (RMSEA), and Akaike's Information Criterion (AIC). In addition, all path coefficients were indicated by standardized estimated values.

\section{RESULTS}

Table 1 presents descriptive statistics for the individual stats: PTS, FGM, FGA, FTA, DR, and FD were significantly higher in the high group than in the other two groups, and were significantly higher in the middle group than the low group. There were no significant differences in FG\%, FTM, FT\%, OR, AS, TO, and BS between the middle and high groups, but these items were significantly higher in these two groups than the low group. There were no differences in ST and PF between the low and middle groups, but ST and PF were significantly higher in the high group than the other two groups. There were no significant differences in PT among the groups.

Figure 1 shows the play model for the high group. Items in category 1 (items directly connected to the score: FGM, FGA, FTM, FTA and AS) had significant causal relationships with TS, except for FTA. In addition, players with more FGM also had more FD. In category 2 (items to increase score opportunities: OR, DR, ST, and BS), OR had a causal relationship with TS. Recording many ORs resulted in an increase in FGA. In category 3 (items to reduce opponents' score opportunities: TO and PF), there was a causal relationship between TO and TS. And recording many TOs resulted in an increase in PF. The model fit indices indicated the model was a good fit (CFI=0.960, RMSEA=0.075, AIC=113.709).

Figure 2 shows the play model for the low group. In category 1 (FGM, FGA, FTM, FTA, and AS), there was no significant causal relationship with TS. In category 2 (OR, DR, ST, and BS), DR and ST showed a causal relationship with TS. In addition, increased ST resulted in an increase in FGA, AS, and FD. In category 3 (TO and PF), TO showed a causal relationship with TS. Recording many TOs resulted in an increase in PF. The model fit was also good (CFI $=0.905$, RMSEA $=0.089$, $\mathrm{AIC}=152.803$ ).

We could not develop a suitable model for the middle group. 
Table 1. Descriptive statistics for individual stats

\begin{tabular}{lccc}
\hline & \multicolumn{3}{c}{ Group $($ Mean \pm SD) } \\
\cline { 2 - 4 } & Low & Middle & High \\
$(\mathrm{n}=124)$ & $(\mathrm{n}=88)$ & $30.3 \pm 6.2$ \\
\hline PT & $28.9 \pm 6.2$ & $29.2 \pm 7.2$ & $13.7 \pm 7.0^{*,+}$ \\
PTS & $2.9 \pm 3.0$ & $9.1 \pm 8.5^{*}$ & $6.1 \pm 3.2^{*,+}$ \\
FGM & $1.3 \pm 1.4$ & $4.1 \pm 3.9^{*}$ & $14.3 \pm 4.9^{*,+}$ \\
FGA & $3.8 \pm 3.4$ & $9.1 \pm 6.0$ & $43.1 \pm 18.0^{*}$ \\
FG\% & $31.3 \pm 31.9$ & $41.6 \pm 24.0^{*}$ & $1.4 \pm 1.5^{*}$ \\
FTM & $0.3 \pm 0.6$ & $0.9 \pm 1.6^{*}$ & $2.6 \pm 2.4^{*,+}$ \\
FTA & $0.6 \pm 1.2$ & $1.6 \pm 2.3^{*}$ & $37.5 \pm 36.9^{*}$ \\
FT\% & $10.8 \pm 24.7$ & $32.0 \pm 41.0^{*}$ & $1.4 \pm 1.3^{*}$ \\
OR & $0.8 \pm 1.0$ & $1.3 \pm 1.2^{*}$ & $6.3 \pm 4.0^{*,+}$ \\
DR & $1.7 \pm 1.4$ & $3.2 \pm 2.8^{*}$ & $3.6 \pm 3.4^{*}$ \\
AS & $1.8 \pm 2.3$ & $3.7 \pm 3.1^{*}$ & $2.5 \pm 1.8^{*,+}$ \\
TO & $1.2 \pm 1.3$ & $1.8 \pm 1.8^{*}$ & $1.4 \pm 1.3^{*,+}$ \\
ST & $0.5 \pm 0.8$ & $0.8 \pm 1.2$ & $0.4 \pm 0.6^{*,+}$ \\
BS & $0.1 \pm 0.3$ & $0.2 \pm 0.5^{*}$ & $2.0 \pm 1.3^{*,+}$ \\
PF & $1.5 \pm 1.3$ & $1.5 \pm 1.3$ & $3.0 \pm 2.1^{*,+}$ \\
FD & $0.8 \pm 1.1$ & $2.0 \pm 2.0^{*}$ &
\end{tabular}

${ }^{*} \mathrm{p}<0.05$ vs. low group, ${ }^{+} \mathrm{p}<0.05$ vs. middle group.

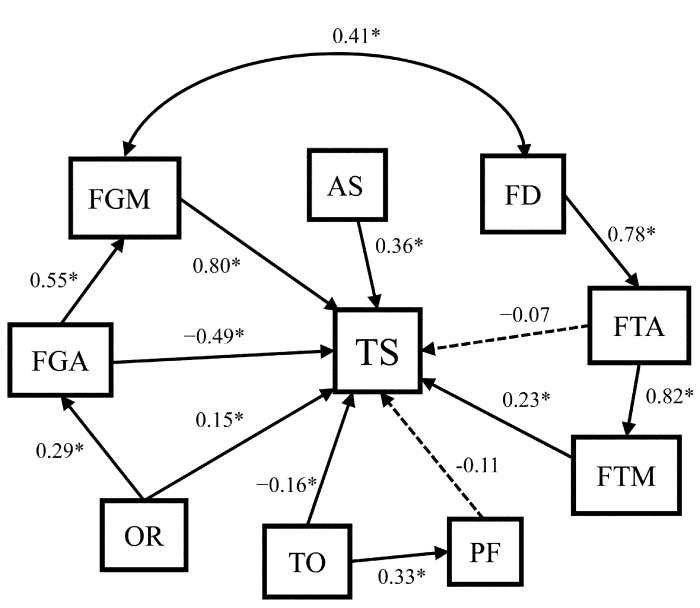

Fig. 1. The high group's scoring play contribution model. $\mathrm{CFI}=0.960, \mathrm{RMSEA}=0.075, \mathrm{AIC}=113.709, * \mathrm{p}<0.05$.

Paths show the influence of the item.

Dotted lines show no significant difference $(\mathrm{p}>0.05)$.

TS: the team's total score; FGM: field goal made; FGA: field goal attempts; FTM: free throw made; FTA: free throw attempts; AS: assist; OR: offensive rebound; TO: turnover; PF: personal foul; FD: draw a foul.

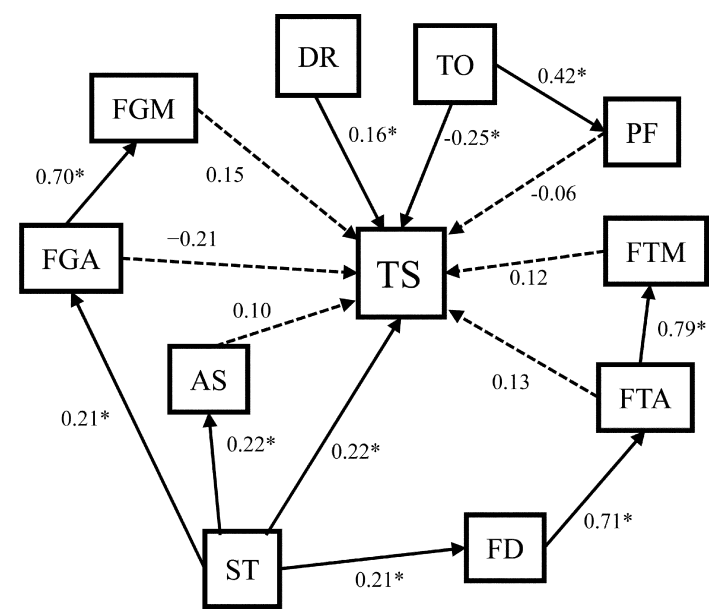

Fig. 2. The low group's scoring play contribution model. $\mathrm{CFI}=0.905, \mathrm{RMSEA}=0.089, \mathrm{AIC}=152.803, * \mathrm{p}<0.05$.

Paths show the influence of the item.

Dotted lines show no significant difference $(p>0.05)$.

TS: the team's total score; FGM: field goal made; FGA: field goal attempts; FTM: free throw made; FTA: free throw attempts; AS: assist; DR: defensive rebound; ST: steal; TO: turnover; PF: personal foul; FD: draw a foul.

\section{DISCUSSION}

Comparison of the stats for each group showed that high scores were recorded for many stats items for players in the high group. As many of these stats are based on ball play, this shows that players classified in the high group often made more direct plays in relation to the ball. This suggests that there is greater reliance on players in this group (who have more trunk function and freedom of movement). This is consistent with results obtained by Vanlandewijck and colleagues ${ }^{2}$, and supports the conclusion that the performance of elite female wheelchair basketball players depends on functional ability. However, 
if too much reliance is placed on a particular player, the opposing team will also concentrate on that player. This means that how play is shared is important in wheelchair basketball. Therefore, even if the stats for players in the low group are lower than those in the high group, their contribution is not necessarily low.

We developed models of play for each group using covariance structure analysis, and analyzed each group's contribution to the team's TS. FGM, FTM, and AS were items directly linked to the TS; however, in the low group, there was no significant causal relationship between these items and TS, whereas there were significant relationships between TS and TO, DR and ST. This shows that players in the low group made less contribution to play directly related to the score, but contributed to the team's TS by increasing ST and DR and decreasing TO, thereby increasing score opportunities. In the high groups, there were strong causal relationships between the team's TS and FGM, FTM and AS. This shows that in offense, greater scoring ability is required for players in the high groups compared with the low group. However, as the standardization estimation value of FGA was negative, determining a shot with good probability is as necessary for success as many shots. In addition, while OR was important in the high group, DR was important in the low groups; even in the same rebound, there was a difference between the high group and the low group. Acquisition of the rebounding ball is advantageous to the defensive side that is often in the inner area. The offensive side requires to invade their opponent's area with contact play to acquire the rebound and winning ball possession; this may be related to trunk function. TO was another important item detected in the high group. As mentioned, players in the high group engage in many plays with the ball, meaning TO influences the team's score.

We could not develop a suitable model for the middle group. This result seems to be because the roles of the middle group, which is between the high group and the low group, differ depending on the team and individuals.

Because there was no difference among the groups in terms of game time, it may be assumed that differences among the groups in the aggregated items were not due to playing time, but were caused by the performance.

This study had some limitations. We only used data for elite female players at the 2016 Rio Paralympic Games; the individual circumstances of players in each country were not considered. In the future, it may be possible to analyze male and female games at various levels and evaluate different game tactics in the change of the age and analyze focusing on specific teams. In summary, in women's wheelchair basketball at the 2016 Rio Paralympic Games, plays more directly involving the ball were associated with players in the high group. These players are required to have high scoring ability, obtain OR, and reduce TO. Key contributions of players in the low group are to increase ST and DR, and suppress TO.

\section{Conflict of interest}

None.

\section{ACKNOWLEDGEMENT}

We thank Audrey Holmes, MA, from Edanz Group (www.edanzediting.com/ac) for editing a draft of this manuscript.

\section{REFERENCES}

1) International Wheelchair Basketball Federation: Official Player Classification Manual. http://www.wheelchairbasketball.ca/uploadedFiles/Members/Classifiers/Policies_and_Procedures/CLASSIFICATION\%20MANUAL\%202014-2018\%20ENGLISH\%20FINAL.pdf\#search=\%27Classification+manual+IW BF\%27 (Accessed Jun. 26, 2017)

2) Vanlandewijck YC, Evaggelinou C, Daly DJ, et al.: The relationship between functional potential and field performance in elite female wheelchair basketball players. J Sports Sci, 2004, 22: 668-675. [Medline] [CrossRef]

3) Cavedon V, Zancanaro C, Milanese C: Physique and performance of young wheelchair basketball players in relation with classification. PLoS One, 2015, 10: e0143621. [Medline] [CrossRef]

4) Vanlandewijck YC, Evaggelinou C, Daly DD, et al.: Proportionality in wheelchair basketball classification. Adapt Phys Activ Q, 2003, 20: 369-380. [CrossRef]

5) Sampaio J, Drinkwater EJ, Leite NM: Effects of season period, team quality, and playing time on basketball players' game-related statistics. Eur J Sport Sci, 2010, 10: 141-149. [CrossRef]

6) Choi DH, Kim SM, Lee JW, et al.: Winning factors: How players' positional offensive and defensive skills affect probability of victory in the Korea Basketball League. Int J Sports Sci Coaching, 2015, 10: 453-459. [CrossRef]

7) Sampaio J, Janeila M, Ibanez S, et al.: Discriminant analysis of game-related statistics between basketball guards, forwards and centres in three professional leagues. Eur J Sport Sci, 2006, 6: 173-178. [CrossRef] 